\title{
Compassion Fatigue Versus Compassionate Care
}

\author{
Corrie Berk, Beth Reefer, Linden Wu and Rose E. Constantino
}

University of Pittsburgh School of Nursing, 3500 Victoria Street, Victoria Building, Pittsburgh, PA 15261, United States

\begin{abstract}
The purpose of this paper is to inform the reader of the signs, symptoms and consequences of Compassion Fatigue (CF) and the various ways it is displayed. Although they are not readily seen by the untrained eye, the informed trained healthcare provider could observe insidious gradual lessening of compassion over time. We present applicable evidence based literature, examine protective factors and interventions, and make recommendations concerning CF. The case study that is presented in a soliloquy is an example of a nurse's story (thinking, feelings and behaviors) devoid of CF while caring for a sexually assaulted patient in an ED and the patient's response to the compassionate care she received. In conclusion, it is important to inform healthcare providers of the ubiquity of stress as well as selfawareness while the provision of social support to staff a top priority for leadership in organizations. Ways this can be done is through open dialogue about coping during orientation sessions and meetings, making resources available to staff, offering crisis debriefings, and emphasizing the positives of patient care, the inexpensiveness of compassionate care and the expensiveness of CF. Staff along with administrators in a healthcare setting should celebrate successful encounters and interventions.
\end{abstract}

Publication History:

\section{Introduction}

The healthcare system staff especially nurses in the Emergency Department [1] are on the front lines to provide emergent care to patients who survive violent crimes. They are at a great risk for compassion fatigue. The purpose of this paper is to inform the reader of the various ways compassion fatigue is displayed although they are not readily seen by the untrained eye; an insidious gradual lessening of compassion over time. The case study we present is an example of a nurse's story while caring for a sexually assaulted patient in an $\mathrm{ED}$ and the patient's response to the compassionate care she received. Furthermore, we share with the reader applicable evidence-based literature, examine protective factors and interventions, and make recommendations concerning compassion fatigue.

Compassion fatigue (CF) is well-documented among healthcare providers, family caregivers, and first responders. It is also presented in the literature by many names: secondary survivor, secondary traumatic stress, vicarious traumatization, and secondary victimization. CF is described as the emotional residue or strain of exposure to working with those suffering from the consequences of traumatic events; may occur due to exposure to one case or to a cumulative level of traumatic events and can be illustrated as running on an empty or close to empty compassion fuel tank; soon alarm bells will ring but no one hears them (Figure 1) [2].

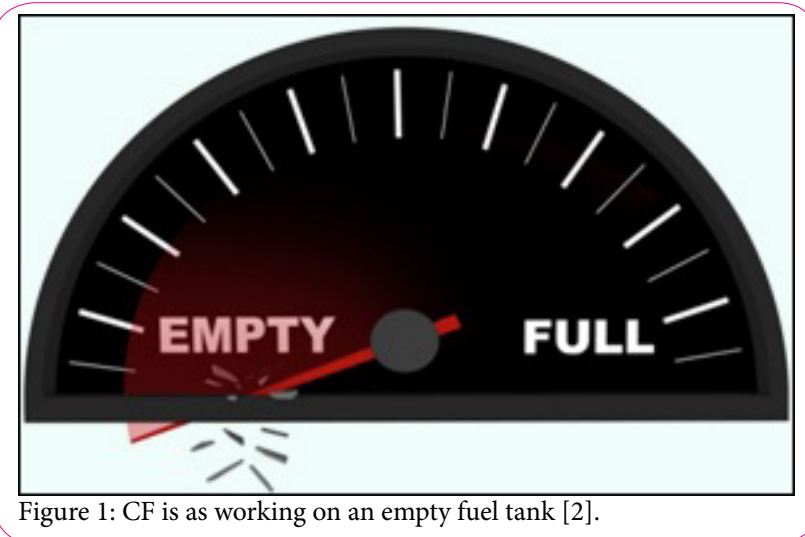

\section{Review of the Literature}

Received: August 22, 2016

Accepted: December 01, 2016

Published: December 03, 2016

\section{Keywords:}

Compassion Fatigue, Emergency

Department, Protective factors,

Traumatic stress,

Posttraumatic stress symptoms,

Compassionate nursing care
Recent research specific to compassion fatigue in the forensic nurse is scarce. While studies specific to the Sexual Assault Nurse Examiner (SANE) are reviewed in this paper, other very recent articles are mentioned as well because they can be applicable to other nursing practice in various settings: trauma, emergency department, first responders, and other developed and developing countries. Sawe[3] wrote on compassion fatigue in emergency providers: experiences from Sub-Saharan Africa as a vignette of an emergency provider in the Sub-Saharan Africa (SSA) setting intertwined with a literature review of CF. The concept paper is a call for help and understanding. $\mathrm{CF}$ is largely studied in the Western countries and neglects countries where there is a disproportionate amount of disease and injury and the burden of caring for them falls on the "trained" and "learned" few.

$\mathrm{CF}$ among emergency care providers in high- and middle-income countries is much more recognized and its protective factors are exclusive to those areas. Protective factors in high-income countries include increased emergency medicine experience, higher levels of information and education, shorter shifts, and adequate social support (peer review, mentorships, etc.) at work. However, these studies do not translate to lower income countries such as the SSA. Studies on CF simply do not exist in areas that lack basic supplies, safe drinking water, and personal protective equipment. Additionally, SSA has high temperatures and humidity as well as high morbidity and mortality especially in their pediatric population. However, CF is no respecter of geography or country. CF can occupy any unsuspecting healthcare provider.

"Corresponding Author: Prof. Rose E. Constantino, University of Pittsburgh School of Nursing, 3500 Victoria Street, Victoria Building, Pittsburgh, PA 15261, United States; E-mail: rco100@pitt.edu

Citation: Berk C, Reefer B, Wu L, Constantino RE (2016) Compassion Fatigue Versus Compassionate Care. Int J Nurs Clin Pract 3: 213. doi: https://doi. org/10.15344/2394-4978/2016/213

Copyright: @ 2016 Berk et al. This is an open-access article distributed under the terms of the Creative Commons Attribution License, which permits unrestricted use, distribution, and reproduction in any medium, provided the original author and source are credited. 
Understanding CF is appropriate to any nurse as s/he may be placed in traumatic environments and emergent situations. Given the divergent nature of the work environment, the nurse may be placed in a situation of practicing "guerilla"-type healthcare where resources and supplies are not ample and conditions are poor. Moving forward in research, it is important to support healthcare providers in these settings and target research of CF in these underserved areas in order to provide real, timely and appropriate effective interventions to combat this costly intrusive but preventable phenomenon.

Berg and others [4] studied CF and burnoutin a trauma setting. Their qualitative study measured compassion fatigue and burnout syndrome in a trauma team consisting of 12 practitioners by allowing them to share perceptions of related stress triggers and coping strategies through surveys and focus groups. More than half of the trauma team scored at risk forcompassion fatigue and burnout syndrome. Stress triggers were described as situation (abuse, age of patient) versus injury-related as well as administrative conflict being largely contributory. Although the sample size of the team was small at only 12 members, the results are consistent with existing literature on CF. Personal coping mechanisms were most often reported.

A noteworthy aspect of Berg and others' study is that data showed the trauma team members were not as adept at managing work stressors as well as they perceived themselves to be. In addition, the lack of institutional resources for coping was a concern. Although first responder teams are often required to attend debriefings after direct exposure to trauma, this may not be the case for those who experience it indirectly and repeatedly that result in familiarity and indifference. Thus, internal mechanisms to advocate for team coping may be necessary as well as better staff support systems in general. The lesson that can be applied from this qualitative study is that the nurse is likely to be affected by the traumas s/he witnessed and vicariously absorbed.

Research with and of forensic nurses on the occurrence of posttraumatic stress symptoms and their relationship to professional quality of life (ProQoL) in nursing staff at a forensic psychiatric security unit[5] is a valuable one. Violence is frequent towards nurses in forensic mental health hospitals but implications of this high risk environment have not been systematically assessed. This study explored the occurrence of post-traumatic stress symptoms (PTSS) and their relationship to professional quality of life for 100 forensic psych nurses. Self-report questionnaires (PCL-C and ProQOL) assessing symptoms of post-traumatic stress and professional quality of life were distributed among these psychiatric nurses in a high security forensic psychiatric unit with high frequency of violent behavior. During the year of the study,staff experienced 221 incidents of threat and violence corresponding to 13.8 incidents/bed/year of which 7.4 incidents/bed/year were physical attacks on staff members [5].

Furthermore, in this same [5]study, relationships between posttraumatic stress symptoms (PTSS), forensic nursing experience, type of ward and compassion satisfaction, burnout and compassion fatigue were assessed. Results from the 70 surveys returned, was a low prevalence of post-traumatic stress symptoms, despite the violence and $96 \%$ of the nurses meeting exposure criteria. This could be explained in several ways. A number of traumatized staff members could have had experienced symptoms of PTSS without being detected in the study because of its cross-sectional design. Some could have had decreased symptoms, some could have left work because of symptoms and are among those not responding to the questionnaire. Some nurses could have had more severe symptoms and been reluctant to answer because of the hallmark avoidance and denial issues related to PTSS.

The low prevalence could perhaps be explained by the special characteristics of the clinic where this study was performed, such as very high patient/staff ratio (1:5), which together with a generally strong collegial spirit within the wards and a strong sense of mutual experience throughout the unit could have contributed to the low frequency of PTSS. Additionally, since the staff is still "in the trenches," they have not been able to re-experience or avoid events as they regularly are exposed to new violent traumatic conflicts. The emotional distance between personnel and patients necessitated by a high security ward could possibly also reduce the emotional impact of violence towards nurses and other staff but may also be viewed as compassion fatigue in and of itself.There were definitely low scores on compassion satisfaction. Interestingly, the length of psychiatric nursing experience and low scores on compassion satisfaction were correlated to PTSS.

It could be speculated that experienced staff withPTSS tend to stay at a ward with high structure and security and less need for engagement in patients' rehabilitation. The long and seasoned experience of these forensic psychiatric nurses leads both to a higher exposure to violence, and therefore to higher rates of post traumatic problems, and to a "natural briefing" of the staff, so that nothing is unexpected, and therefore fewer things are potentially traumatizing. Another key study was Tabor's [6] concept analysis on vicarious traumatization. The author provided a concept map and discussed vicarious traumatization (VT) as well as related terminology, symptomology, prevention and relevant interventions. Suggestions and strategies for personal and professional growth for nurses especially forensic nurses working with victims of violence were provided. This article echoes that the effects of stress on professionals including various negative symptoms that may mirror the biopsychosocial effects exhibited by the victims of trauma. The author argues that multiple concepts including burn out, compassion fatigue, post-traumatic stress disorder (PTSD), and secondary traumatic stress are terms that have been incorrectly interchanged with the term vicarious traumatization (VT). Clarity of VT and understanding its contributing factors is imperative in order to facilitate future research and implement timely and effective interventions, as well as sculpt evidence-based practice [6].

Last but not least is Beck's [7] systematic review of the literature on secondary traumatic stress in nurses. Seven studies in which researchers examined secondary traumatic stress in nurses were analyzed. The samples in five of these studies consisted of all nurses, whereas in the remaining two studies, nurses were included but the results were not specifically reported for the subgroup of nurses. All seven studies revealed that the presence of secondary traumatic stress was reported by forensic nurses, emergency department nurses,oncology nurses, pediatric nurses, and hospice nurses. Three different instruments that measured secondary traumatic stress in practitioners who care for traumatized populations were used: Secondary Traumatic Stress Scale, Compassion Fatigue Self Test for Helpers, and the Compassion Fatigue Scale Revised. Unfortunately, due to the lack of a standardized tool, the studies could not be compared as if one standardized tool was used. Nonetheless, presence of secondary traumatic stress in nurses was reported in all of the studies included in this literature review.

In this systematic literature review by Beck [7], Townsend and 
Campbell's [8] study was included that examined the correlates of secondary traumatic stress among sexual assault nurse examiners (SANEs). The random sample consisted of 110 forensic nurses from SANE programs. Directors of each program were asked to choose the most experienced nurse in the program to participate in the study. The sample consisted of mainly females (99\%) and Caucasians (97\%), and almost half the sample (43\%) held bachelor's degrees. The average number of years the nurses worked as a SANE was 5.16 years. Secondary traumatic stress was measured using Figley's[9] Compassion Fatigue Self-Test (CFST). One fourth of the forensic nurses scored above the cutoff on the 10-item CFST, which was indicative of experiencing some secondary traumatic stress symptoms after sexual assault examinations. Variables related to higher levels of secondary traumatic stress were higher organizational support, more diffuse goals, greater prosecution orientation, and higher caseloads. The authors pointed out that the results of higher organizational support being related to higher secondary traumatic stress was puzzling and offered the explanation that the fit of the path analysis model could have been underestimated by the small sample size. Furthermore, there was no power analysis done in this study. Protective variables against secondary traumatic stress included peer support, satisfaction with compensation, SANE-only facilities, older age, and more education. Although some may have been equating "older age" with "increased experience," this result seems to differ from the Lauvurd and others'[5]study where increased experience correlated with increased PTSD symptoms or increased CP signs.

\section{Causes and Consequences of Compassion Fatigue}

$\mathrm{CF}$ is defined as "an extreme state of tension and preoccupation with the suffering of those being helped to the degree that it is traumatizing for the helper"[9].A strong identification with helpless, suffering, or traumatized people or animals is possibly the motive. It is common for such people to hail from a tradition of what leading traumatologist, Eric Gentry labels: "other-directed care giving." Simply put, these professionals are people who were taught at an early age to care for the needs of others before caring for their own needs. Authentic, ongoing self-care practices may be absent from their lives. And, as Gentry suggests, people who are attracted to caregiving often enter the field already compassion fatigued. It should be noted that compassion fatigue differs from burnout as burnout is defined by exhaustion form the chronic tedium of a job [6].

Caring for others may have a personal cost for the forensics nurse and other healthcare professionals alike. The sequelae of helping others who are traumatized or suffering can result in CF [10]. Healthcare providers need to be aware of strategies that can reduce the impact of working with suffering both on an individual as well as organizational level. A supportive and caring working environment [11] as well as diet, exercise, personal relationships and spiritual support are strategies that individuals can focus on to reduce the symptoms of CF [10]. Without proper support, CF worsens and becomes more detrimental to the individual and the organization in which the individual is involved.

Symptoms generally associated with CF include withdrawal from others, avoidance of intimate relationships, decreased empathy and devaluing of others, outbursts with little provocation, heightened irritability, flashbacks of traumatic events, and hopelessness [12]. More specific observable signs and symptoms associated with compassion fatigue include changes in appetite, sleep and mood disturbances, as well as excessive working, difficulty making decisions, and increase in gastrointestinal upsets and headaches $[13,14]$. Over time, the healthcare provider suffering CF is at risk for depression, anxiety, sleep difficulties, and relational conflicts [11].

As CF remain unattended or ignored, decline in personal job performance and efficiency, a rise in errors and sick time, and a disruption in the morale of the immediate work environment may result [15]. This will ultimately affect the larger system at an organizational level. According to the Compassion Fatigue Awareness Project, when CF hits a critical mass in the workplace, the organization itself will suffer from chronic absenteeism, spiraling Worker's Comp costs, high turnover rates, friction between employees, and conflict between staff and management are among organizational symptoms that surface. This creates additional stress on employees thereby perpetuating the cycle.

In the end, patients ultimately suffer. CF can cause severe depersonalization in making CF sufferers avoid or dread certain patients or certain "types" of patients. This prevents them from developing a therapeutic relationship with patients that is conducive to proper assessment, planning, intervening, and evaluating requirements for a comprehensive and effective nursing care, developing patient trust, providing proper family/caregiver support and creating a safe and comfortable milieu. In the case of nurses at the bedside such as intensive care units or acute care settingsspecifically, negative or disagreeable attitudes could prevent patients from asking for help when needed, compromising patient safety thus ultimately contributing to poorer outcomes.

Early nurse leaders viewed compassion as a fundamental quality of a nurse [16] but the advancements in technology have beenlinked to the decline in the caring nature of some nurses. There are concerns that some nurses are more learned in applying their skills to machines and technical devices, rather than providing compassionate individualized care to patients [17]. One could be suffering from CF if errors are high and job performance is poor, preoccupation about work or specific patients, trouble sleeping and awakening, feeling weary and listless, disengaging in fun activities, and feeling less satisfied, less energetic and less efficient [2].

The soliloquy below is an example of a compassionate nursing care devoid of compassion fatigue. Compassionate nursing care is not merely an emotional and interpersonal connection that the nurse (in this case that follows)designed with the patient but a moral virtue that gives context and direction to the nurse's decisions and actions [18].

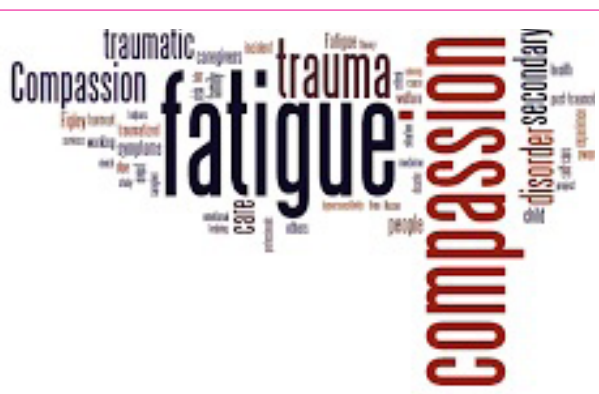

Figure 2: Causes, Consequences and Other Terms and Concepts Used Describe CF. 


\section{A Case of Compassionate Care Devoid of CF}

Every 107 seconds another American is sexually assaulted [19], that's more than 30 people every hour. Over 150 people were attacked during the time we edited this paper. Each year there are nearly 300,000 victims of sexual assault, and many of those rapists are still walking our streets. Despite the efforts law enforcement and the criminal justice system, fewer than 6,000 of these rapists will ever spend a day in prison paying for their crimes [19]. The statistics are mind boggling, making it easy to become overwhelmed and lose hope that anything can be done to suppress this epidemic of violence. On the contrary, I believe there are little things anyone can do. Kofi Annan summed it up perfectly when he said, "Knowledge is power. Information is liberating. Education is the premise of progress, in every society, in every family". This case is written from two perspectives: that of a sexual assault patient-survivor and the SANE nurse who volunteered to care for the patient. No one chooses nor deserves to be violated in this way, but the way in which the situation is handled in the hours after the assault is incredibly life changing for everyone involved.

It's cold. Why is it so cold? God, my head hurts. What happened last night? Okay, pull it together, just focus. I'm so cold. Where are my clothes? How did this happen? I thought I could trust him. I told him "no" so many times. Did I lead him on? My best friend Ann just warned me that I flirt too much. This is my fault. I'm such an idiot. What now? Okay. Get up. Pull yourself together. My whole body hurts. My eye is bruised. I have to work tomorrow. Howam I going to cover this up? I need help. Where's my cell phone? Who can I even call? Pam will just say, "I told you so". Mom will freak out; I can't tell her. I'm all alone. Okay, that's fine, no one needs to know. You can do this alone; just get yourself to the hospital before you pass out again. I want to shower. I want to sleep. Why am I still cold? Okay. What first? Think, Ann, think. Don't shower; that's bad right? Shower is what I so desperately need destroys the evidence right? Right, just get in the car. Drive. It'll all be a bad memory soon. God, this is my fault; just drive. Just get to the hospital and everything will be okay.

A compassionate ED nurse (Alex) noticed Ann immediately, sitting frozen in the corner of the ED, arms wrapped tightly across her chest, shooting terrified looks at the strangers around her. Anyone can recognize that she has just been assaulted. Why hasn't she been triaged yet? Probably for the best; triage can be rough and lengthy. Regardless, victims of sexual assault take priority and are never to be left alone. But this person was left alone cold and disheveled. I'll let the charge nurse know that I'll take care for this patient. She'll have to cover my other patients.

Alex refreshed her memory of her training as a Sexual Assault Nurse Examine (SANE) in a soliloquy. Okay, remember my training: stay calm, never judge the victim, choose gentle and soothing words, avoid all insensitive language, never doubt or question the victim, and do not rush anything above all listen as the patient tells her story. Good, the patient (Ann) appears to be relieved to not be alone anymore; I'll just guide her gently into exam room 10 . This is my favorite exam room in these situations because it is far away from the usual chaos of the ED and is totally private. It is also the only exam room with a door, which is essential. She'll feel much safer here, I can tell. The tension has already started to leave her face. There's already a rape kit in one of the cupboards. When I first got hired here years ago, the hospital had never even heard of a rape kit. It's funny how things change. I now have every room stocked with a kit consisting of a large paper sheet, small paper bags, cotton-tipped swabs, small cardboard boxes, a comb, paper napkins, filter paper, envelopes, red and purple topped tubes for blood sample collection, a speculum, sterile saline, consent forms, documentation forms, discharge information and easy to read instructions. I'll bet not many people realize how detailed and regimented this examination is. If consent is not obtained during each step of the investigation, if a step is missed during the process or even if the evidence is left unattended for a second, it is possible that the evidence can be deemed inadmissible during trial, if the patient chooses to prosecute.

Alex reads Ann's nonverbal response to the care she provides. Ann seems to say: Alex's presence and calmness is comforting. I didn't expect to have to sign consent forms. I figured that once I got to the hospital all my choices would be made for me. It is nice to finally have some control in this situation. So far things seems simple enough...just a bunch of questions about me; it reminds me of giving a medical history at my doctor's office. Oh, no, Alex is asking about the details of the rape. I don't want to do this anymore. I don't think I can do this alone. I should have called my mom. The questions are so specific and embarrassing. After describing every painful, upsetting detail, including the fact that I had been drinking, I'll bet Alex will blame me. But instead she is assuring me that nothing about this situation is my fault. This process is exhausting. It has been only an hour, but it feels like I've been here all day. I don't know why, but I can't stop apologizing. I'm sorry that I didn't go to the hospital sooner. I'm sorry that I was drinking. I'm sorry that I have no money. I'm sorry I brushed me teeth this morning. I must be so annoying. But Alex never changes. She is constantly comforting and reassuring me. She is telling me that we have 48 to 72 hours from the time of the assault to perform this exam, collect viable evidence. Alex informed me about the victim compensation funds that can help me pay for everything. For the first time in 24 hours I feel safe.

Ann, her name is Ann. That's my best friend's name. It's hard to meet these patients without them affecting me. I've been a SANE nurse for almost 7 years, and I remember every one of my patients. There have been too many. Before I began to have my monthly counseling sessions, I carried and internalized each patient with me and I wasn't able to cope with my job and everyday life. Psychological debriefings are allowing me to focus and provide appropriate care to each of my new patients, including Ann. I can see that she is visibly relaxing as I sit with her and run through the list of questions. I understand completely that this has to be so uncomfortable for her, but I know that gaining her trust is one of the most important parts of this process. With all of the initial questions finally out of the way, I can begin to describe each step of the physical examination to Ann.

Through the years, I have developed my own system, starting with the least invasive and most nonthreatening portion of the exam. This includes obtaining a full set of vital signs (blood pressure, temperature, heart rate, and respiration rate) as well as examining the eyes, ears, nose and throat. I will take a swab of the oropharynx to test for gonorrhea and semen. I can recover sperm up to six hours after an assault, even if the victim brushes her teeth or uses mouthwash. As I listen to the heart, lungs, and abdomen with my stethoscope, I am assessing for any bruises, lacerations, scratches or bite marks. I have to make sure that any of Ann's injuries are documented carefully and photographed. If I can get her to be comfortable with me, I'll perform an internal exam. I'll lubricate my speculum with only saline to prevent the spermicidal actions of other lubricants. Carefully I am swabbing her cervix to obtain cultures. I know that non-motile sperm can be detected more than 72 hours after the assault, so I will prepare 
a wet mount. Lastly, I'm going to perform a rectal examination to assess for masses, tearing, or tenderness. I'll swab the rectum for sperm and also test for Chlamydia and gonorrhea.

I know that this exam is going to be very uncomfortable for Ann, especially since she was just raped. I'm hoping she doesn't refuse certain parts of the exam, but if she does, that's okay. She has the right to refuse or stop the process at any time. Evidence collection does not take precedence over her voice; the product of her thinking, feeling and behaving.

I'm zoning out while she is describing the physical exam to me. All of a sudden I'm not in the hospital anymore. I'm back at my house with him holding me down, forcing himself on me. I wish I had been stronger. I wish I could have fought back. What's wrong with me? My mind snaps back into focus as she hands me a fresh hospital gown and asks me to place my clothes in a brown paper bag. A girl who calls herself Bridgett appears suddenly in the room. She must have snuck in during my flash back. I can't let my mind wander back to last night anymore; I promise myself to never revisit those memories again. Somehow I already know I'm going to let myself down. Bridgett is there to hold my hand and comfort me while Alex completes the physical exam. Looking back, I vaguely recall agreeing that she can act as my chaperone.

Whatever, Bridgette seems nice enough, but she doesn't make eye contact with me; is she supposed not to remember me, not to know me for privacy and anonymity? She's probably disgusted. I can't really blame her. I'm disgusted too. I'm zoning out again. Thankfully my mind is just blank. I'll let Alex do whatever she needs to collect the best evidence. I can feel a rage in my soul like nothing I've ever felt before. I want Derek to pay. I want him to hurt like I am hurting right now. The flashes of the camera hardly register as I envision all the ways to punish my rapist... nothing seems enough. I'm looking down at a tourniquet that is placed around my arm. I can't even feel it. I hate giving blood; it makes me queasy, but I can't even feel it. I'm not sure when it happened, but I have stopped shivering. I'm not cold anymore but still numb. I guess the heated blanket Alex gave me before the exam does not help with numbness only the cold.

Ann is doing fine. I can tell she wasn't really present for the exam though; she seems to have separated herself; she watching me perform the exam on her battered body as she watches from afar. She jumps slightly every time I touch her, but otherwise, she stares blankly into space, her face void of emotions. It's common for survivors to have flashbacks after an assault, so it is very important that I approach her slowly and explain everything, even if my words aren't registering. Once the exam is complete I provide her with a clean set of clothes. I'm glad I have arranged with a local department store to give me a discount so that every survivor who enters these ED doors receive new, uncontaminated clothes.

While Ann dresses we discuss treatment of the prevention of pregnancy as well as prophylaxis for sexually transmitted diseases. Options include Ovral and a variety of antibiotics. She refuses any prescriptions but promises that she will follow up with her OB/GYN within the next week. Pretest HIV counseling is a discussion that every SANE nurse must have with his or her patient, so I'll make sure Ann understands. Treatment is made on a case-by-case basis, and all benefits weighted against lifestyle changes, cost, and potential drug toxicity. As I walk Amanda out of the room to prepare for her discharge, I can see the relief in her face. My heart breaks, because I can tell that she thinks the trauma is over, but I know that it truly has just begun.
It's over. The worst of it is over. The "evidence" is collected and bagged. Now I can just put all of this behind me and move on. I think I'm going to keep it to myself. I don't want anyone to know. I'm embarrassed, and no one is going to understand anyway. No one is even going to believe me. Alex gave me some extra clothes to wear home. I didn't expect that. I wonder out loud if they are just leftovers from another patient; clothes that were supposed to be thrown away, until someone had the great idea to given them to the "rape girl". Alex corrects me immediately and says that she buys them herself so that her patients can have something clean and comfortable to go home in. I completely forget she is standing beside me. I'm leaving the hospital as a changed woman...I can feel it already. I try to bite my nails, a habit my mom hates. I forgot Alex trimmed my nails during the assessment. I chew aimlessly on my cuticle instead. I watch her carefully. She's packing a bag for me. I have no idea what she's throwing in there, but I already know I'm just going to throw it all in my closet and never look at it again. Whatever...I grab the bag. The sooner I can get out of this place the better.

Another rape exam completed. It's exhausting for Ann and me. A lot of my patients confess that at first they thought the physical exam was going to be the worst part of the experience. They realize later that it is the physical, emotional and psychological recovery that brings about the most challenges. I try to talk to all my patients about the psychological sequelae of sexual assault, but by the time I am preparing to discharge them, they are usually too overwhelmed and emotionally drained to register any more of my information. Most people don't realize that rape trauma syndrome is similar to posttraumatic stress disorder (PTSD), as nurses like me could develop compassion fatigue $(\mathrm{CF})$

While everyone deals with sexual assault differently, there are generally two phases of recovery. During phase one, the patient experiences a period of fear, anxiety, disbelief, guilt and mood swings. Phase two is a stage of adjustment, integration, and finally recovery. The effects of sexual assault are felt long after the initial insult. Victims of rape are three times more likely to suffer from depression, six times more likely to suffer from post-traumatic stress disorder and twenty six times more likely to abuse drugs. Nearly fifty percent of patients will experience depression at some point during the first year following the attack, and 100 percent report that the experience continues to affect their lives five years later. With these statistics looming in the back of my head, I provide Ann with the appropriate contact information and encourage her to open up to someone close to her about everything that is going on. I also give her my contactinformation on paper in my own handwriting. Survivors of a sexual assault who perceive the physician and nurse to be concerned, kind and organized in their care are far more likely to return for follow-up counseling and treatment. I watch as Ann walks out of the hospital. I say a little prayer that I have done my job well. I hope I see Alex again.

I wave goodbye to Alex as I walk out of the hospital. I refuse to look back, because I worry that I'll never leave. I felt strangely comfortable and safe in that emergency room. I wish I can stay there tonight and every night. Watching Alex work, so organized and structured, but the entire time she never made me feel like I was anything other than her top priority. I am a survivor of sexual assault; survivor is an acceptable term than a rape victim. I am NOT a victim. I am a new person. I never wanted to be this person, but here I am. Alex told me that I cannot let this attack define me, but instead I need to define the attack. I'm still not really sure what that even means, but it sounds 
empowering so I'll go with it. I finally make it to my car and I throw the hospital bag in the front seat. I pick up my cell and dial my mom. I hold my breath while the phone rings. I have no idea what I'm going to say or where to start, but I have to try. As I drive away, I catch a glimpse of the hospital in my rearview mirror. I think one last time about Alex my compassionate nurse and everything she has done for me, without a scintilla of CF. I hope I see her again not in this condition but in a better one.

\section{Implications}

Literature and practice point to numerous implications for the forensic nurse. First, the term compassion fatigue must be appropriately defined and differentiated from PTSD, secondary traumatic stress, vicarious traumatization, and burnout. Through greater education collaboration and advocacy, this is probably the easiest task moving forward. More difficult aspects of defining and differentiating $\mathrm{CF}$ from others include increasing prevention and intervention randomized controlled trials specific to forensic nursing using mixed methods design in data collection and data analyses. Thus far, the literature has divulged numerous CF protective factors, preventive measures, and interventions, but none have truly been put to the test through randomized controlled trials. Similarly, we must ensure that the studies completed have appropriate power analyses and occur in settings most forensic nurses are found or better yet have forensic nurses as study participants.

Additionally, training programs for forensic nurses should revolve around the three levels of prevention: primary before any signs of CF appears or during orientation make $\mathrm{CF}$ a content/learning module for presentation, discussion, and roleplay; secondary early signs and symptoms of CF provide information on descriptions of what are "others"' thoughts, feelings and actions when suffering CF; emphasize what "others" feel and not the person/nurse you are offering secondary prevention. Secondary prevention can include actual referral to intervention services; tertiary post secondary prevention comes tertiary, a follow-up on what has been prescribed during the secondary prevention period provide social support and some rehabilitative period. Here a video or roleplay on definition, differentiation, comparison and contrasting of CF from vicarious traumatization and burnout. Training needs to accentuate positive coping strategies while minimizing negative coping. Recognition of agility and resilience as well as implementation of mirroring techniques could also mitigate occurrence the negative side effects on the forensic nursing career [6]. Perhaps professional organizations such as the American Nurses Association and the International Association of Forensic Nurses are great resource.

From a career planning perspective, the finding of low compassion satisfaction and length of experience predicting PTSD as well as CF symptoms raise questions for how long nurses should work fulltime in these high frequency violent or traumatic environments[5]. If these results are further corroborated in the literature, perhaps career planning over time should include reduced exposure to work with forensic patients, offering other duties such training and mentoring or acknowledging a reduced retirement age such as is the case with police officers and firemen. Fletcher [2] suggested self-awareness as the first and single most important factor in preventing CF. She offered several strategies to cope with CF including but not limited to exercising, maintaining a personal/family life, keeping a sense of humor, setting boundaries between work and home activities, developing your own social support, and broadening your network.

\section{Conclusions}

Forensic nurses are human and not immune to job-related trauma and stress. We need to take care of each other as well as other members of the professional family as much as we take care of patients. Moving forward, it is important to educate healthcare providers of the ubiquity of stress as well as selfawareness and make social support a top priority for leadership in organizations. Ways this can be done is through open dialogue about coping during orientation sessions and meetings, making resources available to staff, offering crisis debriefings, and emphasizing the positives of patient care, the inexpensiveness of compassionate care and the expensiveness of CF. Staff should celebrate successful encounters and interventions.

On an individual level, the best advice is to be kind to yourself. Enhance your awareness with education and accept where you are on your path at all times. Clarify your personal boundaries and make note of what works for you and what doesn't while expressing your needs verbally. It is important to remain proactive, taking positive action to change your environment, instead of being reactive. A good foundation for this is engaging in health-building activities such as exercise, massage, yoga, meditation as well as eating healthy foods and drinking plenty of water. Furthermore, it is important to practice the art of self-management and being able to say "no," reserving your energy for worthy causes. Lastly, it is important to develop a healthy support system made up of individuals that contribute to your self-esteem, listen well, and care.Forensic nurses enter their profession aiming to care for others but they must be equipped to take care of themselves and their peers. We owe it to each other to educate, advocate, and research for building evidence based data to understand, recognize and intervene in CF.

\section{Competing Interests}

The authors declare that they have no competing interests.

\section{References}

1. National youth risk behavior survey overview (2009) Centers for Disease Control and Prevention.

2. Fletcher S (2012) Five strategies to help cope with compassion fatigue. Scrubs: The Nurse's Guide to Good Living.

3. Sawe HR, Murray B, Jamieson J (2016) Compassion fatigue in emergency providers: Experiences from SubSaharan Africa. Emergency Medicine Australasia 28: 109-111.

4. Berg GM, Harshbarger JL, Ahlers-Schmidt CR, Lippoldt D (2016) Exposing compassion fatigue and burnout syndrome in a trauma team:a qualitative study. J Trauma Nurs 23: 3-10.

5. Lauvrud C, Nonstad K, Palmstierna T (2009) Occurrence of post traumatic stress symptoms and their relationship to professional quality of life (ProQoL) in nursing staff at a forensic psychiatric security unit: a crosssectional study. Health Qual Life Outcomes 7: 31.

6. Tabor PD (2011) Vicarious traumatization: Concept analysis. J Forensic Nurs 7: 203-208.

7. Beck CT (2011) Secondary traumatic stress in nurses: a systematic review. Arch Psychiatric Nurs 25: 1-10.

8. Townsend SM, Campbell R (2009) Organizational correlates of secondary traumatic stress and burnout among sexual assault nurse examiners. $J$ Forensic Nurs 5: 97-106. 
Citation: Berk C, Reefer B, Wu L, Constantino RE (2016) Compassion Fatigue Versus Compassionate Care. Int J Nurs Clin Pract 3: 213. doi: https://doi. org/10.15344/2394-4978/2016/213

Page 7 of 7

9. Compassion fatigue: An expert interview with Charles R. Figley, MS, PhD.

10. Absolon $P$, Krueger $C$ (2008) Compassion fatigue nursing support group in ambulatory care gynecology/oncology nursing. in Oncology Nursing Forum. 2008. Oncology Nursing Soc 125 Enterprise Dr, Pittsburgh, Pa 15275 Usa.

11. Stewart DW (2009) Casualties of war: Compassion fatigue and health care providers. Medsurg Nurs 18: 91-94.

12. Gilmore $\mathrm{C}$ (2012) Compassion fatigue-what is it, and how can you avoid it. Nurs N Z 18: 32.

13. Flemister B (2006) Be aware of compassion fatigue. Journal of Wound Ostomy \& Continence Nursing 33: 465-466.

14. Vaughn D (2005) Wounds of war touch nurses, Nursing Spectrum.

15. McHolm F (2006) Rx for compassion fatigue. Journal of Christian Nursing 23: $12-19$.

16. Schantz ML (2007) Compassion: a concept analysis. Nurs Forum 42: 4855 .

17. Knowlden V (1998) The communication of caring in nursing: Sigma Theta Tau International.

18. Von Dietze E, Orb A (2000) Compassionate care: a moral dimension of nursing. Nursing Inquiry 7: 166-174.

19. RAINN (2009) Who are the victims?. 